\title{
Post-Infectious Irritable Bowel Syndrome, an Inflammation-Immunological Model with Relevance for Other IBS and Functional Dyspepsia
}

\author{
Kok-Ann Gwee, FRCP, Ph.D. \\ Yong Loo Lin School of Medicine, National University of Singapore and Gleneagles Hospital, Singapore
}

This review presents studies that support an inflammation-immunological model for the pathogenesis of post-infectious irritable bowel syndrome (IBS), and highlights recent studies that support a similar disease model in non-post-infectious IBS, in particular, diarrhoea-predominant IBS, as well as in post-infectious functional dyspepsia. These recent studies are highlighted to demonstrate that one line of research in functional gastrointestinal disorders has moved away from the old psychosomatic concepts. It is hoped that this will encourage future students of this field to explore the role of immunological events.

(J Neurogastroenterol Motil 2010;16:30-34)

\section{Key Words}

Irritable bowel, Functional dyspepsia, Infection, Inflammation, Immunology

\section{Introduction}

The irritable bowel syndrome (IBS) is a condition that afflicts $10-20 \%$ of the population with a variable combination of abdominal pain, bloating, constipation, and diarrhoea. To patients, scientists and doctors unfamiliar with recent research, it is a condition with no known cause, no animal models, no biomarkers, and no effective treatment. Perhaps because of this, IBS, as well as other functional gastrointestinal disorders, are often perceived to be psychological disorders. However, recent studies on a subset of patients who develop IBS following an episode of gastro- intestinal infection, has allowed us to articulate an inflammation-immunological model that has now been applied as well to other groups of IBS and also to functional dyspepsia. In this review, I will first present the research in post-infectious IBS that supports this model, and then draw attention to recent research that has implicated similar pathogenic factors in non-post-infectious IBS and functional dyspepsia.

\section{Post-Infectious IBS (PI-IBS)}

Studies of PI-IBS have provided us with one of the best working model for understanding the aetiopathogenesis of IBS.

Received: November 19th, 2009 Accepted: January 4th, 2010

(c) This is an Open Access article distributed under the terms of the Creative Commons Attribution Non-Commercial License (http://creativecommons. org/licenses/by-nc/3.0) which permits unrestricted non-commercial use, distribution, and reproduction in any medium, provided the original work is properly cited.

*Correspondence: Kok-Ann Gwee, FRCP, Ph.D.

Adjunct Associate Professor of Medicine, Yong Loo Lin School of Medicine, National University of Singapore, and Gleneagles Hospital, Annexe Block 05-37, 6A Napier Road, Singapore 258500

Financial support: None.

Tel: +65-6-474-6848, Fax: +65-6-475-8285, E-mail: slbclinic@gmail.com

Conflicts of interest: None. 
In the early studies from Sheffield, patients with acute gastroenteritis were followed prospectively to document the development of IBS and to identify predisposing factors. Stress, as represented by the presence of recent traumatic life events, and a neurotic personality trait, were found to be the best predictors of who might develop IBS post-infection (Table 1). ${ }^{1}$ At 3 months post-infection, both, patients who had developed IBS and patients who were asymptomatic, demonstrated accelerated colonic transit and increased rectal sensitivity compared with uninfected controls, with the IBS patients having more severe disturbances. ${ }^{2}$ Rectal biopsies were obtained from a number of patients to study inflammatory markers. Comparing PI-IBS patients with post-infection controls and healthy non-infection controls, we found that IBS patients had relatively greater mucosal cellularity which we interpreted as an indication of a subcolitic inflammatory process. To further support a pathogenic role for inflammation, we demonstrated increased expression of pro-inflammatory cytokine interleukin $1 \beta \mathrm{m}$ RNA in rectal biopsies taken at 3 months post-infection in PI-IBS patients which was not observed in post-infection patients without IBS. ${ }^{3}$ Putting all these data together we proposed a bio-psychophysiological model for PI-IBS. An episode of infectious diarrhoea may produce changes in the gastrointestinal physiology that predispose to the development of IBS symptoms like diarrhoea and pain. However, the manifestation of IBS develops when psychological factors exist in association with more severe inflammatory and physiological disturbances. This could explain why only a fraction of patients with infection develop IBS. Conversely, this could also be used to explain why the majority of patients with psychiatric disturbances do not complain of IBS symptoms.

Studies from other centres support this dynamic and interactive model. Spiller's group from Nottingham studied a group of patients with Campylobacter jejuni gastroenteritis. ${ }^{4}$ Serial rectal mucosal biopsies taken post-infection demonstrated increased counts of intra-epithelial lymphocytes and enteroendocrine cells at 2 weeks, that gradually normalised over 12 weeks, but appeared to persist in patients with PI-IBS. Furthermore, PI-IBS patients had significantly elevated $\mathrm{CD}^{+}{ }^{+}$and $\mathrm{CD} 8{ }^{+}$lymphocytes. In these post-Campylobacter patients, intestinal permeability was also observed to be increased at 6 weeks and 12 weeks post-infection, even in some patients who were asymptomatic. In a subsequent study from Nottingham, it was reported that the intestinal permeability of PI-IBS subjects was increased in the proximal small intestine compared with controls, but this was not as severe as diarrhoea predominant IBS without a history of infection. ${ }^{5}$ In another series of PI-IBS, Marshall and colleagues from Ontario, Canada, studied a cohort of patients involved in a large waterborne outbreak of gastroenteritis, and found increased intestinal permeability in those who developed IBS compared with non-IBS controls. ${ }^{6}$ In animal models, stress has been demonstrated to increase intestinal permeability with enhanced trans-epithelial passage of luminal antigens. ${ }^{7}$ In a recent study women exposed to moderate background stress (in the form of recent life events), demonstrated a defective jejunal epithelial response (increased albumin permeability) to stimuli (cold pain stress) that could predispose them to gut mucosal inflammation (increased luminal tryptase and $\alpha$-defensins release). ${ }^{8}$

A study from China followed a large cohort of patients who had presented with Shigella dysentery between April and October 1998, and their family members who had not suffered dysentery. ${ }^{9}$ Biopsy specimens were taken from the terminal ileum and rectosigmoid regions. In post-Shigella IBS patients increased IL1 $\beta$ $m$ RNA expression was observed in the terminal ileum and rectosigmoid. Interestingly, they also found increased numbers of mast cells in biopsies taken from the terminal ileum of both PI-IBS as well as non-post infectious IBS (non-PI-IBS) patients, compared with controls. Furthermore, in the samples from these IBS patients, both PI-IBS and non-PI-IBS, they observed an increased density of nerve fibres in the vicinity of mast cell collections. At the same time, in Bologna, Barbara et al. had demonstrated a correlation between abdominal pain and the proximity of mast cells to neurons in the distal colonic mucosa in a non-specific group of IBS patients. ${ }^{10}$ Recently, in a study from Korea involving a small number of PI-IBS patients, increased numbers of enterochromaffin cells, mast cells, and lamina propria $\mathrm{T}$ lymphocytes were also reported in biopsies taken from the rectum. ${ }^{11}$

\section{Non-Post-Infectious IBS (majority diarrhoea-predominant IBS)}

The idea that IBS could be an inflammatory disorder has now been extended to other forms of IBS, and in particular diarrhoea predominant IBS (D-IBS). Chadwick et al, reported that almost $90 \%$ of a group of IBS patients (55\% had D-IBS, 36\% had PI-IBS) showed evidence of immune activation regardless of the type of onset. ${ }^{12}$ The authors found higher numbers of CD25 ${ }^{+}$ cells in IBS patients compared with asymptomatic controls. $\mathrm{CD} 25^{+}$cells are regulatory $\mathrm{T}$ cells that are considered important in the prevention of autoimmunity, as well in controlling inflammatory responses in the gut; their absence in mice leads to 
spontaneous colitis. The increased presence of $\mathrm{CD} 25^{+}$cells in IBS raises the possibility that there is auto- or exogenous antigen challenge in these patients, and that the $\mathrm{CD} 25^{+}$cells are preventing the progression to a more florid inflammatory response. In another study involving D-IBS patients (30\% with PI-IBS) intra-epithelial $\mathrm{CD} 3{ }^{+}$lymphocytes were also found to be increased in jejunal biopsies. ${ }^{13}$ In addition, this group of D-IBS patients also presented evidence of mast cell involvement as evidenced by markedly increased mast cell numbers in the jejunal biopsies as well as higher jejunal fluid tryptase concentrations, when compared with healthy controls. In a study involving a highly selected group of ten severe IBS patients, full thickness biopsy was obtained from the jejunum laparoscopically and examined for and shown to have low grade infiltration of lymphocytes in the myenteric plexus. ${ }^{14}$

There is dynamic evidence of intestinal immune activation in IBS. Ohman et al. from Sweden provided evidence of an augmented cell-mediated intestinal immune response. ${ }^{15}$ Peripheral blood lymphocyte were examined for T-cell expression of integrin $\beta 7$, while colonic biopsies were examined for endothelial expression of mucosal addressin cell adhesion molecule-1 (MAdCAM-1), in samples taken from IBS patients $(60 \%$ D-IBS), ulcerative colitis patients and asymptomatic patients with a negative colonoscopy for other indications. T lymphocytes that have been activated in gut-associated lymph nodes express integrin $\beta 7$ which mediates homing to intestinal mucosa by binding to its endothelial expressed ligand MAdCAM-1. In this study IBS patients had an increased frequency of integrin $\beta 7$ and MAdCAM-1 resembling that seen in patients with ulcerative colitis.

Another study provides evidence of activation of the mucosal innate defence system by studying human defensins which are human antimicrobial protein induced by probiotic microorganisms and pro-inflammatory cytokines. In particular, human beta-defensin-2 (HBD-2) has been reported to be expressed in active intestinal inflammation in ulcerative colitis (UC). In the study by Langhorst, faecal HBD-2 levels were measured by enzyme-linked immunosorbent assay (ELISA), while colonic biopsies were tested for HBD-2 peptides by immunohistochemistry. ${ }^{16}$ Compared with healthy controls, patients with UC and IBS (all of whom had diarrhoea) were found to have elevated HBD-2 faecal levels, and in these patients beta-defensin 2 peptides were demonstrated in colonic epithelial enterocytes. These results support a pro-inflammatory response in IBS patients.

In another recent study, Liebregts et al., examined peripheral blood mononuclear cell (PBMC) production of pro-inflamma-

Table 1. Summary of Inflammatory-Immune Activation Markers in FGID Studies

\begin{tabular}{|c|c|c|}
\hline Post-Infectious IBS & Diarrhoea predominant IBS & Functional dyspepsia \\
\hline 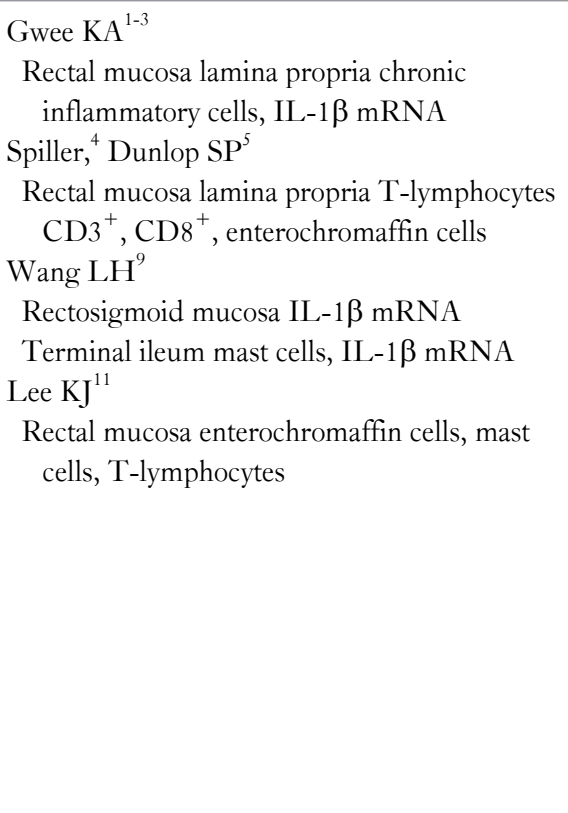 & $\begin{array}{l}\text { Chadwick VS } \\
\text { Colonic mucosa T lymphocyte CD25 } \\
\text { Barbara } \mathrm{G}^{10} \text { (IBS-D 50\%, IBS-C 50\%) } \\
\text { Colonic mucosa mast cells } \\
\text { Guilarte } \mathrm{M}^{13} \\
\text { Jejunal mast cells, intraepithelial lymphocytes }^{+} \mathrm{CD}^{+} \\
{\text {Ohman } \mathrm{L}^{15}} \\
\text { Peripheral blood lymphocytes integrin } \beta 7 \\
\text { Colonic mucosal endothelial MAdCAM-1 } \\
\text { Langhorst J } \\
\text { Fecal HBD-2 } \\
\text { Colonic epithelial enterocytes HBD-2 peptide } \\
\text { Liebregts T } \\
\text { Peripheral blood monocytes TNF } \alpha, \text { IL-1 } \beta \text {, } \\
\text { IL-6, } E \text {. coli LPS induced TNF } \alpha \\
\text { Wang LH }{ }^{16} \\
\text { Terminal ileum mast cells } \\
\text { Lee KJ }{ }^{11} \\
\text { Rectal mucosa mast cells }\end{array}$ & $\begin{array}{l}\text { Hanevik } \mathrm{K}^{23} \text { (Post-Giardia FD) } \\
\text { Duodenal inflammation } \\
\text { Kindt } \mathrm{S}^{25} \text { (Post-infectious FD) } \\
\text { Duodenal T cells, CD }{ }^{+} \text {aggregate } \\
\mathrm{Li} \mathrm{WG}^{26} \\
\text { Gastric mast cells } \\
\text { Talley } \mathrm{NJ}^{27} \text {, Walker MM28 (FD, } \\
\text { post-infection status) } \\
\text { Duodenal mucosal eosinophilia }\end{array}$ \\
\hline
\end{tabular}

IBS, irritable bowel syndrome; FD, functional dyspepsia; LPS, lipopolysaccharide. 
tory cytokines TNF- $\alpha$, IL-1 $\beta$ and IL-6 in IBS patients as a group compared with healthy controls. ${ }^{17}$ Of particular interest was that patients with diarrhoea type IBS, of which PI-IBS formed a substantial number, demonstrated increased basal and Escherichia coli (E. coli) lipopolysaccharide-induced production of TNF- $\alpha$. Furthermore, there was a positive correlation of TNF$\alpha$ production with anxiety scores.

As the production of cytokines is under genetic control, it is possible to study the role of genetics by evaluating the genotypes for various pro- and counter-inflammatory cytokines. Two studies have reported lower frequency in IBS patients of genotypes promoting production of IL-10 which is a counter-inflammatory cytokine. ${ }^{18,19}$ In one study, increased frequency of genotypes that promoted the production of TNF- $\alpha$, a pro-inflammatory cytokine, was reported in IBS patients. ${ }^{19}$ In this study, PI-IBS patients comprised $21 \%$ of the study group; comparing PI-IBS with non-PI-IBS patients the frequency of genotypes for IL-10 and TNF- $\alpha$ appeared similar between the two groups. However, when comparing predominant bowel habits, D-IBS patients had significantly higher prevalence of TNF- $\alpha$ producer genotypes. A recent study employing microarray expression profiling has identified differential expression of genes associated with the host mucosal immune response to microbial pathogens, between IBS patients and healthy controls. ${ }^{20}$

\section{Functional Dyspepsia}

In 2002, an outbreak of Salmonella gastroenteritis occurred in a Spanish village as a result of contamination of cream cakes from a bakery. ${ }^{21}$ When these subjects were followed up prospectively, it was found that Salmonella gastroenteritis was a significant risk factor not only for IBS, but also for functional dyspepsia (FD). Furthermore, a substantial number of subjects had an overlap syndrome of IBS and FD. It was observed that while over time the number of subjects who had dyspepsia alone declined progressively, the number of FD-IBS subjects appeared to remain fairly stable.

A paper from Norway reported the outcome of an outbreak of waterborne giardiasis in Bergen that occurred in $2004 .^{22}$ Out of a total of 1,300 symptomatic cases with Giardia lamblia identified in stool samples, 139 had persistent symptoms despite several treatment courses. An estimated two thirds of these patients had overlapping IBS and FD. Twenty-two post-Giardia infection patients with IBS by Rome II criteria and 19 patients who had recovered from Giardia infection after treatment with metronida- zole, were enrolled into a meat soup gastric emptying study. The post-Giardia IBS patients had lower drinking capacity and slower gastric emptying. In some patients sequential duodenal biopsies demonstrated microscopic inflammation that subsided with time despite the persistence of symptoms. ${ }^{23}$ In a long term follow-up study of 82 patients from this post-Giardia IBS cohort, the authors reported that close to $60 \%$ reported exacerbation of their symptoms by specific foods. ${ }^{24}$

Beyond these two well defined cohorts, studies of what is termed post-infectious functional dyspepsia (PI-FD), are more limited, but nevertheless provides support for an inflammatory and immunological mechanism in the pathogenesis. In a recent study from Belgium, duodenal biopsies were obtained from patients with presumed PI-FD and patients with unspecified FD (U-FD). ${ }^{25}$ Focal aggregates of $\mathrm{T}$ cells and focal $\mathrm{CD}^{+}$aggregates were found in PI-FD but not in U-FD patients. Furthermore, gastric emptying was studied, and found to be delayed in patients with these focal aggregates. In a study from Shanghai, 550 patients with acute gastrointestinal infection were followed-up for half a year, and the prevalence of PI-FD was estimated to be $6.7 \%$ and epigastric pain, epigastric burning sensation, and early satiety were the major symptoms in these patients. ${ }^{26}$ In gastric biopsies from PI-FD patients, the number of activated mast cells, as evidenced by the total amount of tryptase and histamine released in the gastric mucosa, was reported to be higher than non-PI-FD patients and healthy controls. Furthermore, the number of mast cells located within 5 microns of nerve fibers was significantly higher in PI-FD patients than in the other two groups.

In a large study from Sweden, duodenal biopsies were obtained from asymptomatic and symptomatic subjects drawn from a large community cohort offered screening gastroscopy. ${ }^{27,28}$ Insufficient information was provided to determine the proportion of subjects with PI-FD. However, unusually, Helicobacter pylori infection was significantly less common in their FD subjects than subjects with IBS criteria. In their cohort, FD subjects had duodenal eosinophilia compared to asymptomatic controls, and an association with early satiety was reported.

\section{Conclusion}

These recent studies enable us to better conceptualise how immunological events may lead to the development of IBS and FD, and how environmental, genetic and psychological factors modulate the outcome. They also help us to move beyond the old 
paradigm of functional and organic. The hope is that with this more sophisticated approach we can overcome the prejudices associated with the psychosomatic model, and become better at identifying biomarkers, and at developing animal models and more effective treatments, in what were traditionally functional gastrointestinal disorders.

\section{References}

1. Gwee KA, Graham JC, McKendrick MW, et al. Psychometric scores and persistence of irritable bowel after infectious diarrhoea. Lancet 1996;347:150-153.

2. Gwee KA, Leong YL, Graham C, et al. The role of psychological and biological factors in postinfective gut dysfunction. Gut 1999;44: 400-406.

3. Gwee KA, Collins SM, Read NW, et al. Increased rectal mucosal expression of interleukin $1 \beta$ in recently acquired post-infectious irritable bowel syndrome. Gut 2003;52:523-526.

4. Spiller RC, Jenkins D, Thornley JP, et al. Increased rectal mucosal enteroendocrine cells, $\mathrm{T}$ lymphocytes and increased gut permeability following acute Campylobacter enteritis and in post-dysenteric irritable bowel syndrome. Gut 2000;47:804-811.

5. Dunlop SP, Hebden J, Campbell E, et al. Abnormal intestinal permeability in subgroups of diarrhea-predominant irritable bowel syndromes. Am J Gastroenterol 2006;101:1288-1294.

6. Marshall JK, Thabane M, Garg AX, et al. Intestinal permeability in patients with irritable bowel syndrome after a waterborne outbreak of acute gastroenteritis in Walkerton, Ontario. Aliment Pharmacol Ther 2004;20:1317-1322.

7. Killiaan AJ, Saunders PR, Bijlsma PB, et al. Stress stimulates transepithelial macromolecular uptake in rat jejunum. Am J Physiol 1998;275:G1037-G1044.

8. Alonso C, Guilarte M, Vicario M, et al. Maladaptive intestinal epithelial responses to life stress may predispose healthy women to gut mucosal inflammation. Gastroenterology 2008;135:163-172.

9. Wang LH, Fang XC, Pan GZ. Bacillary dysentery as a causative factor of irritable bowel syndrome and its pathogenesis. Gut 2004;53: 1096-1101.

10. Barbara G, Stanghellini V, De Giorgio R, et al. Activated mast cells in proximity to colonic nerves correlate with abdominal pain in irritable bowel syndrome. Gastroenterology 2004;126:693-702.

11. Lee KJ, Kim YB, Kim JH, Kwon HC, Kim DK, Cho SW. The alteration of enterochromaffin cell, mast cell, and lamina propria $\mathrm{T}$ lymphocyte numbers in irritable bowel syndrome and its relationship with psychological factors. J Gastroenterol Hepatol 2008;23: 1689-1694.

12. Chadwick VS, Chen WX, Shu D, et al. Activation of the mucosal immune system in irritable bowel syndrome. Gastroenterology 2002;122:1778-1783.

13. Guilarte M, Santos J, de Torres I, et al. Diarrhoea-predominant IBS patients show mast cell activation and hyperplasia in the jejunum. Gut 2007;56:203-209.

14. Tornblom H, Lindberg G, Nyberg B, Veress B. Full-thickness biopsy of the jejunum reveals inflammation and enteric neuropathy in irritable bowel syndrome. Gastroenterology 2002;123:1972-1979.

15. Ohman L, Isaksson S, Lundgren A, Simren M, Sjovall H. A controlled study of colonic immune activity and $\beta 7^{+}$blood $\mathrm{T}$ lymphocytes in patients with irritable bowel syndrome. Clin Gastroenterol Hepatol 2005;3:980-986.

16. Langhorst J, Junge A, Rueffer A, et al. Elevated human beta-defensin-2 levels indicate an activation of the innate immune system in patients with irritable bowel syndrome. Am J Gastroenterol. 2009; 104:404-410.

17. Liebregts T, Adam B, Bredack C, et al. Immune activation in patients with irritable bowel syndrome. Gastroenterology 2007;132: 913-920.

18. Gonsalkorale WM, Perrey C, Pravica V, Whorwell PJ, Hutchinson IV. Interleukin 10 genotypes in irritable bowel symdrome: evidence for an inflammatory component? Gut 2003;52:91-93.

19. Van der Veek PP, van den Berg M, de Kroon YE, Verspaget HW, Masclee AA. Role of tumor necrosis factor-alpha and interleukin-10 gene polymorphisms in irritable bowel syndrome. Am J Gastroenterol 2005;100:2510-2516.

20. Aerssens J, Camilleri M, Talloen W, et al. Alterations in mucosal immunity identified in the colon of patients with irritable bowel syndrome. Clin Gastroenterol Hepatol 2008;6:194-205.

21. Mearin F, Perez-Oliveras M, Perello A, et al. Dyspepsia and irritable bowel syndrome after a salmonella gastroenteritis outbreak: one year follow-up cohort study. Gastroenterology 2005;129:98-104

22. Dizdar V, Gilja OH, Hausken T. Increased visceral sensitivity in Giardia-induced postinfectious irritable bowel syndrome and functional dyspepsia. Effect of the $5 \mathrm{HT}_{3}$-antagonist ondansetron. Neurogastroenterol Motil 2007;19:977-982.

23. Hanevik K, Hausken T, Morken MH, et al. Persisting symptoms and duodenal inflammation related to Giardia duodenalis infection. J Infect 2007;55:524-530.

24. Hanevik K, Dizdar V, Langeland N, Hausken T. Development of functional gastrointestinal disorders after Giardia lamblia infection. BMC Gastroenterol. 2009;9:27.

25. Kindt S, Tertychnyy A, de Hertogh G, Geboes K, Tack J. Intestinal immune activation in presumed post-infectious functional dyspepsia. Neurogastroenterol Motil. 2009;21:832-e56.

26. Li WG, Li XB, Ge WZ. A preliminary study of acute gastrointestinal infection-associated functional dyspepsia (Article in Chinese). Zhonghua Nei Ke Za Zhi 2008;47:739-742.

27. Walker MM, Talley NJ, Prabhakar M, et al. Duodenal mastocytosis, eosinophilia and intraepithelial lymphocytosis as possible disease markers in the irritable bowel syndrome and functional dyspepsia. Aliment Pharmacol Ther 2009;29:765-773.

28. Talley NJ, Walker MM, Aro P, et al. Non-ulcer dyspepsia and duodenal eosinophilia: an adult endoscopic population-based case-control study. Clin Gastroenterol Hepatol 2007;5:1175-1183. 